\title{
Farinha desmucilada de taro utilizada na elaboração de bolos
}

\section{Demucilated taro corm flour used in cake preparation}

\section{Luan Alberto ANDRADE'; Carina Lumie Pereira NAGATA²; Giovana Maria Pereira ASSUMPÇÃO; Gilma Auxiliadora Santos GONÇALVES ${ }^{3}$; Joelma PEREIRA ${ }^{4}$}

\begin{abstract}
1 Autor para correspondência; Doutorando em Ciência dos Alimentos; Universidade Federal de Lavras - UFLA; Departamento de Ciência dos Alimentos; Laboratório de Grãos Raízes e Tubérculos; Campus Universitário, caixa postal 3037, CEP 37200-000, Lavras MG, Brasil. Tel +55-35-3829-1660 Fax:+55-35-3829-1401; E-mail: luandrade87@yahoo.com.br

${ }^{2}$ Doutoranda em Ciência dos Alimentos; Universidade Federal de Lavras - UFLA; carinanagata@gmail.com

${ }^{3}$ Professoras do Instituto Federal de Educação, Ciência e Tecnologia, Sudeste - MG Campus Barbacena, Doutorandas em Ciência dos Alimentos - Universidade Federal de Lavras - UFLA; giovana.assumpcao@ifsudestemg.edu.br; gilma.goncalves@ifsudestemg.edu.br

${ }^{4}$ Professora Dra. Associada III do Departamento de Ciência dos Alimentos - Universidade Federal de Lavras - UFLA; joper@dca.ufla.br
\end{abstract}

Recebido em: 29-09-2014; Aceito em: 01-03-2015

\section{Resumo}

O bolo é um produto que atende grande parcela de consumidores, portanto, é interessante adicionar ingredientes que melhorem sua qualidade. O objetivo do trabalho foi caracterizar a farinha desmucilada de taro (FDT) e fazer sua aplicação na elaboração de bolos, sem alterar seus atributos tecnológicos e sensoriais. Na FDT, foram realizadas as análises de composição centesimal, açúcares, amido, $\mathrm{pH}$, acidez titulável, fibra alimentar e minerais. Os bolos foram elaborados com diferentes concentrações de FDT (0\%, 5\%, 10\%, 15\% e 20\%) em substituição parcial à farinha de trigo. As análises realizadas nos bolos foram umidade, $\mathrm{pH}$, acidez titulável, performance, volumes, densidade, cor, textura e análise sensorial. A FDT apresentou elevadas concentrações de amido, fibra alimentar e de microminerais. Os parâmetros $\mathrm{pH}$, simetria, encolhimento e taxa de elasticidade dos bolos não apresentaram diferença significativa a 5\% de probabilidade. A acidez titulável, altura média, força de compressão, tonalidade cromática $\left(\mathrm{h}^{*}\right)$ e croma $\left(\mathrm{C}^{*}\right)$ adequaram-se ao modelo quadrático de regressão, e a diferença de cor total $(\Delta \mathrm{E})$ ajustou-se ao modelo linear de regressão. Os demais parâmetros não se ajustaram ao modelo matemático proposto. Quanto à análise sensorial, todas as formulações apresentaram bom índice de aceitabilidade ( $\geq 70 \%)$ para os atributos aparência, sabor, textura e impressão global, com exceção da formulação com $20 \%$ de FDT, que apresentou índice levemente menor para aparência (67\%). A composição química da FDT justifica sua utilização na elaboração de bolos, até $20 \%$ de adição, sem alterações expressivas que desfavoreçam o produto final.

Palavras-chave adicionais: análise sensorial; Colocasia esculenta; fibra alimentar; mapa de preferência interno.

\begin{abstract}
Cake is a product that serves a large portion of consumers and thus it is interesting to add ingredients that improve its quality. The objective herein was to characterize the demucilated taro flour (FDT) and its application in cakes without altering their technological and sensory attributes. Analyzes of proximate composition, sugars, $\mathrm{pH}$, titratable acidity, dietary fiber and minerals were conducted on FDT. The cakes were prepared with different concentrations of FDT $(0 \%, 5 \%, 10 \%, 15 \%$ and $20 \%)$ in partial substitution of wheat flour. Analyzes conducted in the cakes were moisture, $\mathrm{pH}$, titratable acidity, performance, volume, density, color, texture and sensory analysis. The FDT showed high concentrations of starch, dietary fiber and micro-minerals. The $\mathrm{pH}$, symmetry, shrinkage and elasticity insex parameters of the cakes showed no significant difference at $5 \%$ probability. The titratable acidity, average height, compression strength, $\mathrm{h}^{*}$ and $\mathrm{C}^{*}$ fit the quadratic regression model, the $\Delta \mathrm{E}$ adjusted to the linear regression model. The other parameters did not fit the proposed mathematical model. Regarding sensory analysis, all formulations showed a good acceptability index ( $\geq 70 \%)$ for the attributes appearance, flavor, texture and overall impression, with the exception of the $20 \%$ of FDT formulation, which showed a slightly lower index for appearance (67\%). The chemical composition of FDT justifies its use in the preparation of cakes, with up to an added $20 \%$, without significant changes that compromise the final product.
\end{abstract}

Additional keywords: Colocasia esculenta; fiber; food sensory analysis; internal preference mapping. 


\section{Introdução}

O taro (Colocasia esculenta) é uma cultura de rizomas consumida em áreas tropicais (Aboubakar et al., 2008), sendo o décimo quarto alimento vegetal mais consumido no mundo (Nunes et al., 2012). Este vegetal é um membro da Família Arum (Araceae), que pode ser conhecida por diversos nomes, como inhame e taioba (Kaushal et al., 2012), seu caule é modificado em rizoma amiláceo, constituindo a parte comestível da planta (Santos \& Puiatti, 2002). Este rizoma é um importante alimento energético, rico em vitaminas e sais minerais (Leonel et al., 2005), sendo considerado como boa fonte de carboidratos e magnésio (Huang et al., 2007).

A mucilagem do taro desempenha papel de interesse na indústria de alimentos em razão de suas propriedades como espessante, estabilizante e emulsificante (Tavares et al., 2011). Na extração dessa mucilagem, obtêm-se uma farinha fibrosa que possui suas características ainda desconhecidas cientificamente, podendo apresentar grande potencial de aplicação em produtos de panificação, como bolos, biscoitos e pães. Devido ao valor nutricional do rizoma de taro (Franco, 1998), acredita-se que essa farinha obtida possa ser rica em minerais, como ferro, além de possuir fibra alimentar e outros compostos.

O bolo, embora não constitua um alimento básico como o pão, é consumido por pessoas de diferentes faixas etárias (Borges et al., 2006), atendendo a grande parcela de consumidores em geral, o que justifica a utilização da farinha desmucilada de taro (FDT) com finalidade de aumentar o consumo de fibras pela população, sem alterar negativamente atributos como cor, sabor e textura do produto final. $\mathrm{O}$ bolo é um produto obtido pela mistura, homogeneização e cozimento conveniente de massa preparada com farinhas, fermentadas ou não, e outras substâncias alimentícias (como, por exemplo, leite, ovos e gordura) (Borges et al., 2006).

Mudanças no processamento e a crescente exigência do consumidor por alimentos com qualidade sensorial, nutricional e que tragam benefícios à saúde incentivam o estudo de novos ingredientes para a indústria de alimentos (Silva et al., 2009), que poderá ter como exemplo a FDT. A análise sensorial é um método acessível para avaliar as características de diversos produtos, como o bolo, bem como sua aceitação pelo público-alvo, tornando-se, portanto, uma análise interessante quando modifica ou adiciona novos ingredientes em alimentos tradicionais, pois nada adianta adicionar fibras e nutrientes em produtos acabados se eles não possuem boa aceitação pelo público.

Nesse contexto, o objetivo do trabalho foi caracterizar quimicamente a farinha desmucilada de taro (FDT) e sua aplicação em bolos, sem alterar seus atributos tecnológicos.

\section{Material e métodos}

\section{Obtenção da farinha desmucilada de taro (FDT)}

Os rizomas de taro foram obtidos no comércio varejista de hortifrutigranjeiros. Esses rizomas foram lavados em água corrente para eliminação de sujidades superficiais, sanificados por imersão em água clorada com hipoclorito de sódio (200 $\left.\mathrm{mg} \mathrm{L}^{-1}\right)$ por 15 minutos, descascados manualmente, triturados em liquidificador industrial (Lucre, Catanduva, Brasil). Uma porção de massa triturada de taro integral foi pesada e filtrada manualmente em malha de poliéster $(40 \mathrm{~cm} \times 40 \mathrm{~cm})$, obtendo-se um filtrado (mucilagem) e um resíduo (fração retida), sendo a proporção obtida desses componentes em torno de $53,9 \%$ e $46,1 \%$, respectivamente.

O resíduo obtido da extração da mucilagem de taro foi encaminhado à estufa com circulação de ar, a $65^{\circ} \mathrm{C}$, por aproximadamente 72 horas, até peso constante. Após seco, foi moído em liquidificador industrial (Lucre, Catanduva, Brasil), para a desintegração dos grumos e total homogeneização, obtendo-se, portanto, a farinha desmucilada de taro (FDT), com rendimento de aproximadamente 35\%.

\section{Caracterização química da farinha desmucilada de taro (FDT)}

Com a obtenção da FDT, foram realizadas as seguintes análises:

1. Umidade: de acordo com a metodologia $\mathrm{n}-$ 925.09 da AOAC (2000), até a obtenção do peso constante;

2. Extrato etéreo: de acordo com a metodologia no 925.38 da AOAC (2000);

3. Proteína bruta: de acordo com o método de micro- Kjeldahl no 920.87 da AOAC (2000);

4. Cinzas: de acordo com o método gravimétrico da AOAC (2000) № 923.03, com calcinação a $550{ }^{\circ} \mathrm{C}$, com permanência da amostra dentro da mufla-FORNITEC, modelo 1926, Brasil;

5. Fibra alimentar: as amostras em triplicata foram gelatinizadas com Termamyl e, então, digeridas enzimaticamente com proteases e amiloglucosidase para remoção de proteína e amido. Quatro volumes de etanol foram adicionados para precipitar a fibra alimentar. O resíduo total foi filtrado, lavado com etanol a $78 \%$ e $95 \%$ e acetona. Depois de seco, - resíduo foi pesado. Duas amostras foram analisadas para proteína, e a terceira foi incinerada a $525^{\circ} \mathrm{C}$, determinando-se as cinzas. O valor de fibras totais foi igual ao peso do resíduo, menos o peso médio de proteínas mais cinza, de acordo com a metodologia da AOAC (1995);

6. Fração glicídica: foi calculada pelo método de diferença, como determina a AOAC (2000);

7. Valor calórico: foram utilizados fatores de conversão de Atwater, conforme metodologia de Osborne \& Voogt (1978), em que $4 \mathrm{kcal} \mathrm{g}^{-1}$ para proteínas e carboidratos e $9 \mathrm{kcal} \mathrm{g}^{-1}$ para lipídeos. $\mathrm{O}$ resultado foi expresso em kcal $100 \mathrm{~g}^{-1}$; 
8. Açúcares totais, redutores e não redutores: foram analisados segundo o método de Somogy, adaptado por Nelson (1944);

9. Amido: foi extraído por hidrólise ácida, segundo técnica da AOAC (1990) e identificado pelo método de Somogy, modificado por Nelson (1944);

10. Acidez Titulável e $\mathrm{pH}$ : foram determinados de acordo com a metodologia de Cecchi (2003). Sendo a acidez titulável expressa em miliequivalente de $\mathrm{NaOH}$ por 100 gramas da matéria seca;

11. Minerais: utilizou-se da metodologia descrita por Malavolta et al. (1997).

\section{Análise estatística}

Realizou-se a análise estatística descritiva, obtendo-se média e desvio-padrão de três repetições para as análises realizadas na caracterização da farinha desmucilada de taro (FDT).

\section{Elaboração de bolos com diferentes concentra- ções de FDT}

Foram elaborados bolos utilizando as concentrações de $0 \%, 5 \%, 10 \%, 15 \%$ e $20 \%$ da FDT em substituição à farinha de trigo. As formulações utilizadas estão apresentadas na Tabela 1.

Tabela 1 - Formulações dos bolos elaborados com diferentes concentrações de farinha desmucilada de taro (FDT). Cakes formulations prepared with different concentrations of demucilated taro flour (FDT).

\begin{tabular}{lccrrr}
\hline Ingredientes & $0 \%$ & $5 \%$ & $10 \%$ & $15 \%$ & $20 \%$ \\
\hline Farinha de trigo & $270 \mathrm{~g}$ & $256,5 \mathrm{~g}$ & $243 \mathrm{~g}$ & $229,5 \mathrm{~g}$ & $216 \mathrm{~g}$ \\
FDT & - & $13,5 \mathrm{~g}$ & $27 \mathrm{~g}$ & $40,5 \mathrm{~g}$ & $54 \mathrm{~g}$ \\
Margarina & $60 \mathrm{~g}$ & $60 \mathrm{~g}$ & $60 \mathrm{~g}$ & $60 \mathrm{~g}$ & $60 \mathrm{~g}$ \\
Açúcar & $180 \mathrm{~g}$ & $180 \mathrm{~g}$ & $180 \mathrm{~g}$ & $180 \mathrm{~g}$ & $180 \mathrm{~g}$ \\
Ovos & $103 \mathrm{~g}$ & $103 \mathrm{~g}$ & $103 \mathrm{~g}$ & $103 \mathrm{~g}$ & $103 \mathrm{~g}$ \\
Fermento químico & $9 \mathrm{~g}$ & $9 \mathrm{~g}$ & $9 \mathrm{~g}$ & $9 \mathrm{~g}$ & $9 \mathrm{~g}$ \\
Leite & $165 \mathrm{~mL}$ & $165 \mathrm{~mL}$ & $165 \mathrm{~mL}$ & $165 \mathrm{~mL}$ & $165 \mathrm{~mL}$ \\
\hline
\end{tabular}

Os ingredientes, em temperatura ambiente, foram pesados em balança digital (Bel Engineering, Brasil), nas quantidades descritas, e homogeneizados em batedeira elétrica planetária modelo BPA350W (Arno, Brasil). Os ingredientes secos foram misturados primeiro, com exceção do fermento químico, que foi acrescentado por último e, em seguida, adicionaram-se os demais. Posteriormente, a massa formulada pronta foi depositada em uma fôrma retangular de $20,5 \mathrm{~cm} \times 9 \mathrm{~cm}$ e levada ao forno elétrico modelo FE4242BR (Suggar, China) a $180^{\circ} \mathrm{C}$, por 30 minutos.

\section{Caracterização dos bolos}

As análises de umidade, $\mathrm{pH}$ e acidez titulável foram realizadas seguindo metodologia descrita para a FDT.

O volume foi determinado pelo deslocamento de sementes de painço, de acordo com método AACC (2000). O volume específico foi calculado pela divisão do volume sobre massa. Os bolos foram pesados em balança semianalítica, modelo BL3200H (Shimadzu, Kyoto, Japão), e assim foi determinada a densidade pela relação massa e volume.

O desempenho dos bolos foi avaliado segundo a metodologia desenvolvida pela empresa ICL Brasil, citado por Fabri (2012). Foram, portanto, cortadas fatias no sentido transversal em cada bolo, com dois $\mathrm{cm}$ de largura por $19 \mathrm{~cm}$ de comprimento (em média). Logo após, foram realizadas as medições de acordo com a Figura 1. A partir dessas medidas, foram calculados: a) altura média - composta pela média das medidas $\mathrm{B}, \mathrm{C}$ e $\mathrm{D}$. Calculada de acordo com a equação: $(B+C+D) / 3$; b) índice de uniformidade - calculado de acordo com a fórmula: $B$ - D; C) simetria - calculada de acordo com a fórmula: $2 \times C-(B+D) ; d)$ encolhimento - calculado pelo diâmetro interno da assadeira menos o diâmetro médio do bolo frio.

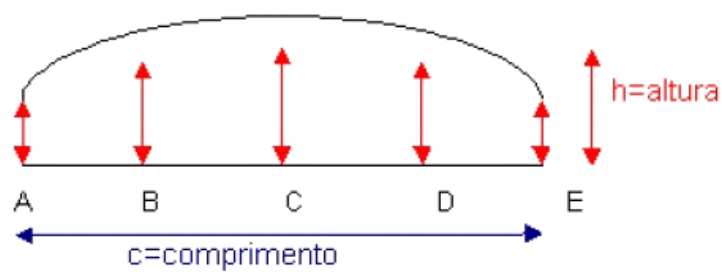

Figura 1 - Medições realizadas para a avaliação do desempenho de bolos (Fabri, 2012). Measurements for evaluating cake performance (Fabri, 2012).

A cor do miolo dos bolos foi determinada utilizando-se de colorímetro modelo CM-5 (Konica Minolta, Osaka, Japão), empregando-se iluminante D65 e geometria 45/0. Os valores de cor foram expressos no sistema CIELAB. Os valores de $L^{*}$ (luminosidade), $a^{*}$ (componente vermelho-verde) e $b^{*}$ (componente amarelo-azul) foram obtidos diretamente do colorímetro e utilizados para o cálculo da tonalidade cromática $\left(h^{*}=\arctan b^{*} / a^{*}\right)$ e do croma ou saturação $\left(C^{*}=\left(a^{* 2}+b^{*}\right)^{1 / 2}\right)$. A diferença de cor total $(\Delta E)$ foi calculada de acordo com a equação: $\Delta \mathrm{E}=\left[\left(\Delta \mathrm{L}^{*}\right)^{2}+\right.$ $\left.+\left(\Delta a^{*}\right)^{2}+\left(\Delta b^{*}\right)^{2}\right]^{0,5}$ (Lawless \& Heymann, 1998)., sendo $\Delta$ a variação de cada uma dessas variáveis.

Para determinar a firmeza e a elasticidade dos 
bolos, foi utilizado o analisador de textura TAXT2i (Stable Micro Systems, Godalming, Inglaterra,). Como sonda, foi empregada a sonda cilíndrica P/36R. Todos os dados obtidos foram analisados utilizando o software Exponent Lite Express (Stable Micro Systems, Godalming, Inglaterra). A temperatura de análise foi de, aproximadamente, $25{ }^{\circ} \mathrm{C}$, e os parâmetros de configuração do aparelho foram: (i) velocidade de teste $=1,0 \mathrm{~mm} \mathrm{~s}^{-1}$; (ii) velocidade de pré-teste $=1,0 \mathrm{~mm} \mathrm{~s}^{-1}$; (iii) velocidade de pós-teste $=10,0 \mathrm{~mm} \mathrm{~s}^{-1}$; (iv) taxa de compressão $=25,0 \%$ de deformação, e tempo de compressão $=60$ segundos.

\section{Análise sensorial do bolo}

O teste de aceitação foi realizado em cabines individuais, obtendo-se sessenta consumidores, de ambos os sexos, com idades variando de 18 a 60 anos. Os participantes eram consumidores regulares de bolos. As cinco amostras de bolos foram servidas em uma sessão, na forma balanceada, para minimizar o efeito da ordem de apresentação e o efeito que uma amostra exerce na avaliação da próxima (Walkeling \& Macfie, 1995), seguindo o delineamento em blocos completos. As amostras foram servidas na temperatura ambiente de, aproximadamente, $25{ }^{\circ} \mathrm{C}$ em recipientes plásticos, inodoros, codificados com números aleatórios de três dígitos. Durante o teste, foi servida água para lavar o palato.

Os bolos foram avaliados quanto à aceitação em relação aos atributos de aparência, sabor, textura e impressão global, utilizando escala hedônica estruturada de nove pontos, variando entre os termos hedônicos: "gostei extremamente" (escore 9) a "desgostei extremamente" (escore 1), e também quanto à intenção de compra por meio de escala de cinco pontos, variando entre os termos: "certamente compraria" (escore 5) a "certamente não compraria" (escore 1).

Para cada um dos atributos avaliados, realizou-se o cálculo do Índice de Aceitabilidade do produto, sendo adotada a equação: $\mid \mathrm{A}(\%)=\mathrm{A} \times 100 / \mathrm{B}$, em que, $A=$ nota média obtida para 0 produto $e$ $\mathrm{B}=$ nota máxima dada ao produto. $\mathrm{O}$ IA com boa repercussão tem sido considerado maior ou igual a 70\% (Meilgaard et al., 1991).

\section{Análise estatística}

O delineamento experimental da segunda etapa foi o inteiramente casualizado (DIC), com cinco tratamentos, em quatro repetições. Os resultados foram analisados por meio de análise de variância, para constatar a significância estatística dos tratamentos. Em seguida, foi realizada regressão polinomial para ajuste de equações matemáticas a $10 \%$ de probabilidade, com base nos coeficientes de determinação (R2) apresentados. Para os dados da análise sensorial, foi realizado teste de média (Tukey) a 5\% de probabilidade e mapa de preferência interno (MPI).

\section{Resultados e discussão}

\section{Farinha desmucilada de taro (FDT)}

$\mathrm{Na}$ Tabela 2, encontram-se as médias e o desvio-padrão da composição centesimal, valor calórico, teores de açúcares totais, redutores e não redutores, amido, $\mathrm{pH}$ e acidez titulável da farinha desmucilada de taro (FDT).

Tabela 2 - Composição química, valor calórico, pH e acidez titulável da farinha desmucilada de taro (FDT). Chemical composition, calorific value, $p H$ and titratable acidity of demucilated taro flour (FDT).

\begin{tabular}{|c|c|}
\hline Caracterização da FDT ${ }^{1}$ & $\begin{array}{c}\text { Média } \pm \\
\text { desvio padrão }\end{array}$ \\
\hline Umidade $\left({\left.\mathrm{g} 100 \mathrm{~g}^{-1}\right)^{\star *}}^{*}\right.$ & $65,03 \pm 0,89$ \\
\hline 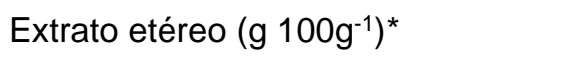 & $0,23 \pm 0,01$ \\
\hline 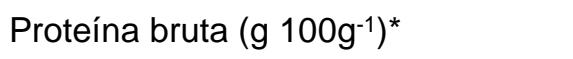 & $2,59 \pm 0,01$ \\
\hline Fibra Alimentar $\left(\mathrm{g} 100 \mathrm{~g}^{-1}\right)^{*}$ & $14,75 \pm 0,02$ \\
\hline Cinzas $\left(g 100 g^{-1}\right)^{*}$ & $3,02 \pm 0,02$ \\
\hline Fração glicídica $\left({\left.\mathrm{g} 100 \mathrm{~g}^{-1}\right)^{*}}^{*}\right.$ & $79,42 \pm 0,03$ \\
\hline Valor Calórico (kcal 100g-1) & $330,07 \pm 0,18$ \\
\hline Açúcar total $\left(\mathrm{g} 100 \mathrm{~g}^{-1}\right)^{*}$ & $8,93 \pm 0,09$ \\
\hline Açúcar redutor $\left(\mathrm{g} 100 \mathrm{~g}^{-1}\right)^{*}$ & $3,68 \pm 0,07$ \\
\hline Açúcar não redutor $\left(\mathrm{g} 100 \mathrm{~g}^{-1}\right)^{*}$ & $4,98 \pm 0,07$ \\
\hline Amido $\left(\mathrm{g} 100 \mathrm{~g}^{-1}\right)^{*}$ & $50,01 \pm 058$ \\
\hline $\mathrm{pH}^{*}$ & $5,78 \pm 0,02$ \\
\hline Acidez Titulável $\left(\mathrm{mEq} \mathrm{NaOH} 100 \mathrm{~g}^{-1}\right)^{*}$ & $6,02 \pm 0,18$ \\
\hline
\end{tabular}

Pode-se observar que o resíduo antes da desidratação possui alto teor de umidade, obtendo-

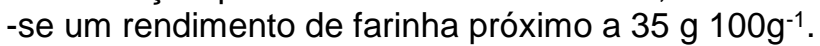

A FDT obtida possui baixo teor de extrato etéreo, devido ao rizoma ser pobre nesse componente. Para Franco (1998), o rizoma de taro possui



Os componentes encontrados neste estudo em maiores concentrações foram: fibra alimentar, fração glicídica e amido, mostrando que esse subproduto é rico em carboidratos, sendo, portanto, uma fonte de energia.

Zárate et al. (2002), trabalhando com farinha de inhame, uma hortaliça semelhante ao taro, no que se refere à composição química, encontraram teor

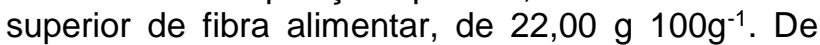
acordo com a ANVISA (Resolução 54, de 12 de novembro de 2012), um alimento sólido é considerado fonte de fibras quando apresenta, no mínimo, $3 \mathrm{~g}$ para cada $100 \mathrm{~g}$ de alimento ou 2,5 g por porção (Brasil, 2012). No caso da FDT, em base seca, possui cerca de 14,75 g a cada $100 \mathrm{~g}$ do produto de fibra alimentar total, sendo, então, interessante sua adição no processamento de alimentos, como, por exemplo, em bolos, pães e biscoitos, pois, 
de acordo com Chavan \& Kadam (1993), que estudaram produtos como "cookies" e bolos, os produtos de panificação são os melhores veículos para incrementar o nível de fibras.

De acordo com Dutra \& Marchine (1998), a fibra alimentar é considerada alimento funcional, pois desempenha funções importantes no organismo, como, por exemplo, intervir no metabolismo dos lipídeos e carboidratos e na fisiologia do trato gastrointestinal, além de proporcionar uma absorção mais lenta dos nutrientes e promover uma sensação de saciedade, o que torna importante sua ingestão em alimentos.

Além de elevado teor de fibra, a farinha desmucilada de taro (FDT) possui alto teor de amido. De acordo com Nip (1997), o amido de taro é considerado altamente digerível, devido ao pequeno tamanho de seus grânulos. Para Huang et al. (2000), a combinação dos grânulos pequenos e o alto teor de fibra alimentar solúvel fazem do rizoma de taro uma boa fonte de carboidratos para fabricação de produtos especiais, tais como para dietas infantis e para idosos, e isto pode ser estendido à sua FDT, conforme as análises realizadas no presente trabalho. Miamoto (2008) encontrou

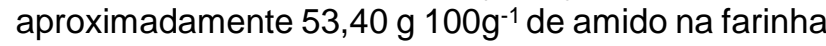
do resíduo de inhame, valor bem próximo ao encontrado no presente trabalho.

Em relação aos teores de açúcares, foram relativamente altos. Andrade (2013) encontrou valores para açúcares totais, redutores e não redutores na mucilagem proveniente do rizoma de taro bem inferiores

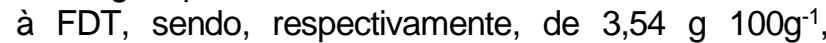

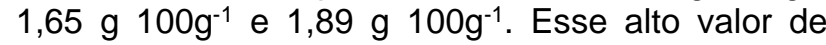
açúcar na FDT pode interferir nas propriedades tecnológicas dos bolos elaborados.

$\mathrm{O}$ valor de $\mathrm{pH}$ foi levemente ácido $(5,78)$, semelhante ao trabalho de Miamoto (2008), que foi de 5,96 . Esses valores de pH estão acima de 4,5, valor que delimita 0 desenvolvimento de microrganismos. Em relação à acidez titulável $(6,02 \pm 0,18 \mathrm{mEq} \mathrm{NaOH}$ $100 \mathrm{~g}^{-1}$ ) da farinha obtida, foi inferior ao trabalho citado anteriormente. Esse fato se deve, provavelmente, à diferença de ácidos orgânicos presentes nas amostras e também à possível diferença no estágio fisiológico do rizoma de taro que obteve a farinha desmucilada. A determinação da acidez em alimentos é importante, haja vista que, por meio dela, podem-se obter dados valiosos na apreciação do processamento e do estado de conservação.

Em relação aos minerais, a FDT apresentou maior destaque para os microminerais ferro, manganês e zinco (Tabela 3), mostrando, portanto, a importância da adição desta farinha no processamento de alimentos, no intuito de enriquecê-los nutricionalmente. O mineral $\mathrm{Fe}$, por exemplo, é muito importante para o organismo, pois ativa enzimas como a catalase, que assegura a degradação dos radicais livres prejudiciais, além de estar envolvido em muitas reações de óxidorredução (Hopkins et al., 1997).
Tabela 3 - Valores de macrominerais e microminerais da farinha desmucilada de taro. Values of macro and trace minerals of demucilated taro flour.

\begin{tabular}{lc}
\hline Macrominerais & $\mathrm{g}^{100 \mathrm{~g}^{-1}}$ \\
\hline $\mathrm{P}$ & 0,12 \\
$\mathrm{~K}$ & 0,93 \\
$\mathrm{Ca}$ & 0,00 \\
$\mathrm{Mg}$ & 0,05 \\
$\mathrm{~S}$ & 0,00 \\
\hline Microminerais & $\mathrm{mg} \mathrm{kg}^{-1}$ \\
\hline $\mathrm{Cu}$ & 3,0 \\
$\mathrm{Mn}$ & 22,4 \\
$\mathrm{Zn}$ & 48,3 \\
$\mathrm{Fe}$ & 47,3 \\
$\mathrm{~B}$ & 3,9 \\
\hline
\end{tabular}

Os macrominerais $\mathrm{P}, \mathrm{K}$ e $\mathrm{Mg}$ apresentaram baixos valores na FDT. Os minerais $\mathrm{Ca}$ e $\mathrm{S}$ não foram encontrados na amostra. De acordo com Ybarra et al. (2001), vários estudos indicam a possibilidade de interação entre cálcio e minerais essenciais, podendo conduzir a uma diminuição na absorção desses nutrientes, consideração feita especialmente para ferro e zinco. O fato de a FDT não apresentar cálcio pode ser considerado fator positivo, uma vez que essa farinha apresenta alto teor de ferro e zinco, impedindo, assim, a competição entre esses minerais durante a absorção no intestino e favorecendo a biodisponibilidade desses microminerais.

\section{Bolos com diferentes concentrações de FDT}

Os valores encontrados para a umidade do bolo não se adequaram ao modelo matemático proposto (regressão polinomial de primeiro $\mathrm{e}$ segundo graus) (Tabela 4). No presente trabalho, todos os bolos com FDT obtiveram percentagem de umidade superior ao controle $(23,21 \%)$, oscilando nas formulações adicionadas de FDT entre $24,80 \%$ e $27,16 \%$. Essa elevação pode ser devida ao aumento de fibras no bolo, proporcionado pela adição da farinha desmucilada, a qual tem propriedade de reter e manter água em sua estrutura durante a cocção. Fato semelhante foi observado por Guimarães et al. (2010), com a substituição da farinha de trigo por farinha de entrecasca de melancia em bolos, sendo identificado aumento no teor de umidade, atribuído também ao acréscimo de fibra.

Não houve diferença significativa, a $5 \%$ de probabilidade, para os valores de $\mathrm{pH}$ dos bolos estudados, sendo a média de 6,39 (Tabela 4). No trabalho de Maia (2007), com o bolo enriquecido de farinha de maracujá $(5 \%)$ e bolo-controle $(0 \%)$, detectou-se o $\mathrm{pH}$ próximo à neutralidade, 6,65 e 7,20, respectivamente, semelhante ao presente trabalho. Para Asm \& Colmey (1973), a faixa ótima de pH de bolos varia entre 6,6 e 7,5, intervalo um pouco superior ao encontrado.

Houve aumento crescente da acidez titulável (Figura 2A) com a substituição da farinha de trigo pela desmucilada de taro (FDT). Resultado semelhante foi 
observado por Guimarães et al. (2010). Esse fato se deve à acidez presente na farinha utilizada e à possível ocorrência da formação de compostos voláteis e com características ácidas durante o processo de cocção.

Tabela 4 - Valores médios e desvio-padrão para os parâmetros umidade, pH e taxa de elasticidade dos bolos formulados com diferentes concentrações da farinha desmucilada de taro. Average values of the parameters index moisture, $\mathrm{pH}$ and the elasticity rate of cakes formulated with different of demucilated taro flour concentrations.

\begin{tabular}{lccc}
\hline Amostras & $\mathrm{U}^{1}(\%)$ & $\mathrm{pH}$ & $\mathrm{TE}^{2}(\%)$ \\
\hline $0 \%$ & $23,22 \pm 0,22$ & $6,37 \pm 0,04$ & $41,50 \pm 1,22$ \\
$5 \%$ & $27,17 \pm 0,38$ & $6,39 \pm 0,05$ & $42,98 \pm 0,59$ \\
$10 \%$ & $25,74 \pm 0,67$ & $6,39 \pm 0,01$ & $41,41 \pm 0,40$ \\
$15 \%$ & $24,81 \pm 0,75$ & $6,39 \pm 0,01$ & $42,50 \pm 1,04$ \\
$20 \%$ & $25,87 \pm 0,36$ & $6,39 \pm 0,02$ & $41,18 \pm 1,10$ \\
\hline
\end{tabular}

${ }^{1} \mathrm{U}:$ umidade; ${ }^{2} \mathrm{TE}:$ Taxa de Elasticidade.

Observou-se decréscimo da altura média dos bolos (Figura 2B) com o aumento da percentagem de FDT quando comparado ao bolo-padrão ( $0 \%$ de FDT).

O índice de uniformidade não se ajustou ao modelo de regressão proposto. Os valores encontrados variaram de 0,08 a $0,30 \mathrm{~cm}$ (Tabela 5). Segundo ICL Brasil (2010), citado por Fabri (2012), o índice de uniformidade permite verificar desníveis na superfície, sendo que quanto mais próximo ao zero, melhor. No presente trabalho, a formulação contendo $5 \%$ de FDT foi a que obteve valor menor $(0,08 \%)$.

Os resultados da avaliação de simetria dos bolos não apresentaram diferença significativa (Tabela 5). O valor médio para este parâmetro foi de $0,45 \mathrm{~cm}$, valor inferior ao encontrado no trabalho de Caruso (2012), que foi de 3,0 a $3,5 \mathrm{~cm}$ e de Schmiele et al. (2011), que obtiveram de 0,55 a $2,28 \mathrm{~cm}$ com incremento de farinha de aveia (0 a 40\%). Segundo ICL Brasil (2010), citado por Fabri (2012), os valores de simetria devem ficar entre $0,30 \mathrm{~cm}$ e $1,0 \mathrm{~cm}$, indicando que houve um leve crescimento no centro do bolo, o que aconteceu com os bolos elaborados. De acordo com Borges et al. (2006), o desenvolvimento simétrico da massa durante o forneamento é uma característica importante no processamento de produtos de panificação. Para bolos, a simetria ideal assume valor zero, indicando crescimento uniforme da massa e manutenção estrutural durante a cocção. A forma ou simetria de produtos de panificação deve ser uniforme e bem definida, caso contrário indica manuseio e processamento inadequados.
A

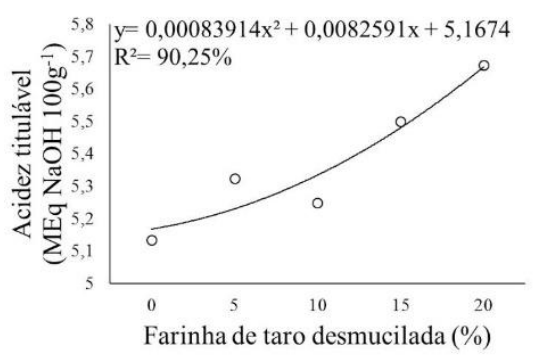

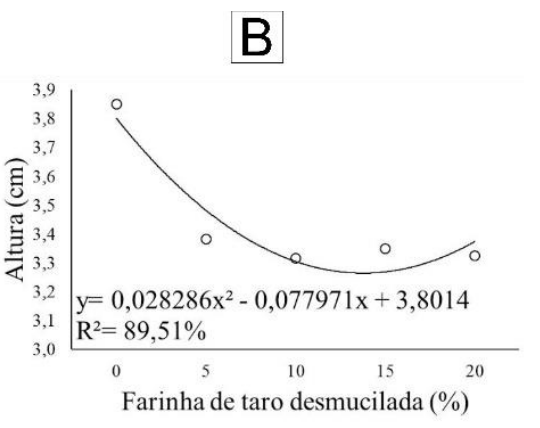

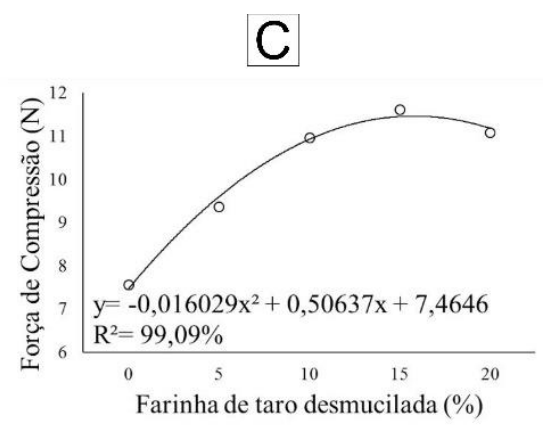

Figura 2 - Modelos de regressão ajustados para: a) acidez titulável; b) altura; c) força de compressão. Regression models adjusted for $A$ ) acidity $B$ ) average height $C$ ) compression strength.

Houve diferença significativa entre as formulações de bolos quanto ao volume do produto pronto, porém não se adequando em nenhum modelo de regressão. Observou-se que, em todos os bolos adicionados com FDT, o volume foi reduzido quando comparados ao bolo-controle, variando entre 525 e $610 \mathrm{~cm}^{3}$ (Tabela 5). Pode-se inferir que a farinha desmucilada interferiu na formação da expansão da estrutura proteica da massa, provavelmente pelo maior conteúdo de fibras. Comportamento semelhante foi observado em estudo desenvolvido por Borges et al. (2006) em bolos elaborados com diferentes concentrações de farinha de aveia, sugerindo que a composição química da farinha de aveia integral interferiu na formação e na agregação da estrutura proteica ao redor das bolhas de ar na massa, contribuindo para a redução do volume do produto final. Guimarães et al. (2010) obtiveram valores menores para o volume dos bolos elaborados com maior concentração (30\%) da farinha de entrecasca de melancia, comparada aos demais tratamentos ( $0 \%$ e $7 \%)$. Tal fato pode ser justificado, uma vez que a adição de fibras causa efeito negativo na qualidade final de produtos panificados. Os efeitos 
mais evidentes são a redução de volume, o aumento da firmeza do miolo, o aparecimento do miolo escuro, e também, em alguns casos, uma modificação do sabor (Wang et al., 2002).

Tabela 5 - Valores médios e desvio-padrão para os parâmetros índice de uniformidade, simetria, encolhimento, volume e densidade dos bolos formulados com diferentes concentrações da farinha desmucilada de taro. Average values of the parameters index uniformity, symmetry, shrinkage, bulk density and the elasticity rate of cakes formulated with different of demucilated taro flour concentrations.

\begin{tabular}{llllllc}
\hline Amostras & ${ }^{(1)} \mathrm{IU}(\mathrm{cm})$ & ${ }^{(2)} \mathrm{S}(\mathrm{cm})$ & ${ }^{(3)} \mathrm{V}\left(\mathrm{cm}^{3}\right)$ & ${ }^{(4)} \mathrm{VE}\left(\mathrm{cm}^{3} / \mathrm{g}\right)$ & ${ }^{(5)} \mathrm{D}\left(\mathrm{g} / \mathrm{cm}^{3}\right)$ & ${ }^{(6)} \mathrm{E}(\mathrm{cm})$ \\
\hline $0 \%$ & $0,25 \pm 0,19$ & $0,30 \pm 0,20$ & $610,0 \pm 21,6$ & $2,8 \pm 0,10$ & $0,35 \pm 0,01$ & $1,18 \pm 0,05$ \\
$5 \%$ & $0,08 \pm 0,21$ & $0,28 \pm 0,24$ & $525,0 \pm 12,9$ & $2,6 \pm 0,10$ & $0,39 \pm 0,01$ & $1,10 \pm 0,14$ \\
$10 \%$ & $0,30 \pm 0,08$ & $0,40 \pm 0,08$ & $545,0 \pm 19,15$ & $2,8 \pm 0,04$ & $0,36 \pm 0,01$ & $1,10 \pm 0,14$ \\
$15 \%$ & $0,15 \pm 0,19$ & $0,45 \pm 0,19$ & $527,5 \pm 9,57$ & $2,7 \pm 0,11$ & $0,38 \pm 0,02$ & $1,10 \pm 0,20$ \\
$20 \%$ & $0,15 \pm 0,13$ & $0,15 \pm 0,13$ & $527,5 \pm 9,57$ & $2,7 \pm 0,10$ & $0,38 \pm 0,01$ & $1,20 \pm 0,00$ \\
\hline
\end{tabular}

(1) IU: índice de uniformidade; (2) S: simetria; ${ }^{(3)}$ V: volume; (4) VE: volume específico; (5) D: densidade; (6) E: encolhimento.

Os resultados dos parâmetros de volume específico e densidade dos bolos avaliados neste estudo apresentaram diferença significativa a 5\% entre si, embora não houvesse ajuste de um modelo de regressão (Tabela 5). O volume específico é a razão entre o volume e a massa do bolo. É um parâmetro de qualidade que indica se a fermentação do bolo foi excessiva, resultando num volume específico muito grande, ou se ocorreram problemas na formação da estrutura do bolo na fermentação, resultando num baixo volume específico (Bushuk, 1985). O volume específico e a densidade evidenciam a relação entre o teor de sólidos e a fração de ar existente na massa assada. Massas com densidade alta ou volume específico baixo, chamadas de embatumadas, apresentam aspecto desagradável ao consumidor. Tais falhas são associadas com a alta umidade, falhas no batimento e na cocção, pouca aeração, difícil mastigação, sabor impróprio e baixa conservação. Neste sentido, os bolos necessitam de quantidade adequada de líquidos, fermento químico, emulsificante e aeração adequados. Massas com grande volume (maior fração ar / sólidos) caracterizam-se por granulometria mais aberta e menor densidade (Esteller et al., 2006).

Quanto ao encolhimento dos bolos, não foi observada diferença significativa entre os tratamentos avaliados, variando entre $1,10 \mathrm{~cm}$ e $1,20 \mathrm{~cm}$ (Tabela 5).

A cor é um aspecto fundamental da qualidade de alimentos, sendo o primeiro atributo observado sensorialmente antes do consumo (Hecktheuer, 1996). Em produtos forneados, a cor é um parâmetro crítico. Os bolos com crostas muito claras ou muito escuras são associados a falhas no processo e na formulação (Esteller et al., 2005). A cor é dada pela matriz $\left(h^{*}\right)$, que é a presença de um comprimento de onda dominante, e também pela saturação (croma), que é a concentração em torno do comprimento de onda dominante (Minolta, 1994). A tonalidade ou matriz $h^{*}$ é expressa em ângulos e inicia em $0^{\circ}=$ vermelho (equivale ao $\left.+a^{*}\right), 90^{\circ}=$ amarelo $\left(+b^{*}\right)$, $180^{\circ}=$ verde $\left(-a^{*}\right)$ e $270^{\circ}$ definido como sendo o azul $\left(-b^{*}\right)$. Os valores de $h^{*}$ observados nos bolos apresentaram comportamento quadrático com redução proporcional ao aumento da adição da farinha desmucilada de taro (FDT) (Figura 3A), com médias variando entre $83,8^{\circ}$ para a formulaçãopadrão e $75,6^{\circ}$ para a formulação com $20 \%$ de FDT, representada pela modificação da tonalidade da cor em relação ao padrão. Marangoni (2007) avaliou a cor de bolos produzidos com farinha de yacon e de linhaça, e observou que os bolos se tornavam mais escuros, conforme maior quantidade dessas farinhas eram incorporadas à massa.

A saturação $C^{*}$, denominada Croma, é um parâmetro associado à pureza da cor de um objeto, sendo "zero" no centro do diagrama, e seu valor aumenta de acordo com a distância do centro, tornando-se mais saturada. Pode-se observar a redução nos valores de $\mathrm{C}^{*}$ dos bolos tanto quanto ao aumento da proporção de FDT incorporada à massa (Figura 3B), evidenciando a diminuição da intensidade da cor dos bolos com maiores teores da FDT. Comportamento contrário foi observado por Marangoni (2007), avaliando formulações que continham maiores teores de farinha de yacon, as quais apresentaram maior saturação, no estudo da potencialidade de aplicação de farinha de yacon em produtos à base de cereais.

Os $\Delta \mathrm{E}$ indicam quanto a amostra diferiu do padrão, e são frequentemente utilizados no controle de qualidade e nos ajustes de formulação, além de serem utilizados para o cálculo da diferença total de cor (Duangmal et al., 2008). Nesse estudo, os valores de $\Delta \mathrm{E}$ dos bolos elaborados com diferentes concentrações de FDT apresentaram aumento linear em relação ao padrão (Figura $3 \mathrm{C}$ ), ou seja, formulações com maiores teores de FDT mostraram maior diferença na cor. Fato semelhante aconteceu no estudo de Borges et al. (2006) ao aumentarem a concentração de quinoa na formulação dos bolos. 

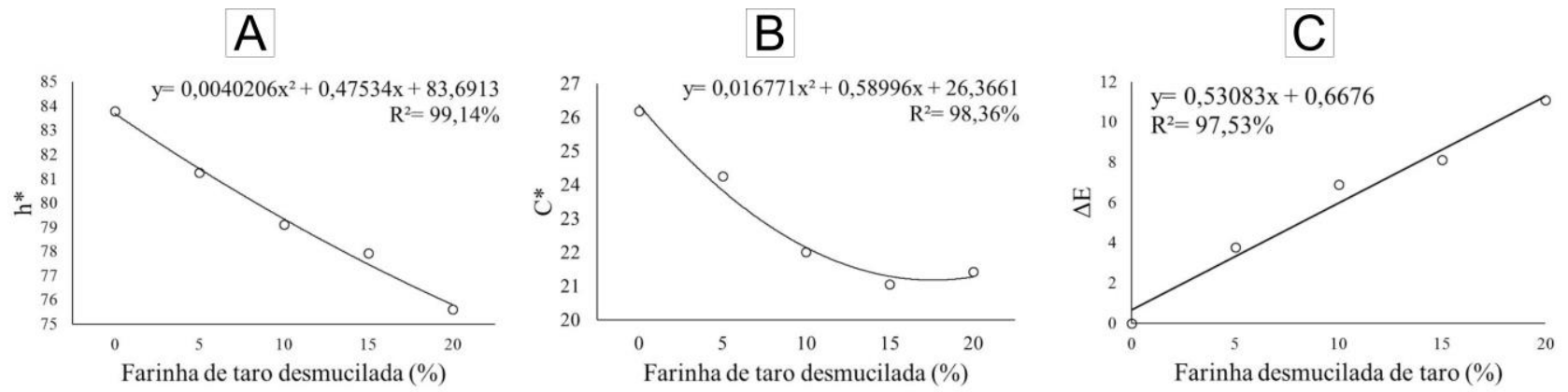

Figura 3 - Valores de cor observados nos bolos e modelos de regressão ajustados para A) $h^{*}$; B) $C^{*}$; C) $\Delta E$. Color values observed in cakes and regression models adjusted by A) $h^{*}$; $\left.B\right) C^{*}$; C) $\Delta E$.

A dureza de bolos avaliada por métodos instrumentais é proporcional à força aplicada para ocasionar uma deformação ou rompimento da amostra e está correlacionada à mordida humana durante a ingestão desse alimento (Esteller et al., 2004). Quanto maior o valor dessa força, maior é a firmeza do produto analisado. Houve diferença significativa quanto aos valores de força de compressão das formulações de bolo em relação ao aumento da concentração da farinha, evidenciando maior firmeza na medida do aumento da adição da farinha desmucilada de taro (FDT) aos bolos, com leve redução entre os valores de 15 a $20 \%$ de adição de FDT (Figura 2C).

Neste estudo, todas as formulações avaliadas apresentaram-se estatisticamente iguais ao padrão quanto à taxa de elasticidade, com valor médio de 41,91\% (Tabela 4).

Ao se desenvolver um novo produto, é fundamental avaliar sua aceitação por parte dos consumidores. Sendo assim, no que se refere à análise sensorial, a Tabela 6 apresenta as notas de aceitação dadas pelos provadores aos bolos formulados com diferentes concentrações de FDT para os atributos aparência, sabor, textura, impressão global e intenção de compra.

Todos os atributos avaliados, por meio da ANOVA, apresentaram diferença significativa, a $5 \%$ de probabilidade, entre os tratamentos.

Com relação à aparência, por meio do teste de Tukey, não houve diferença entre as médias dos bolos formulados com $0 \%$ e $5 \%$ de FDT; este, por sua vez, não diferiu dos bolos de $10 \%$, e este último não diferiu dos bolos de $15 \%$. Os bolos produzidos com $20 \%$ de FDT apresentaram a nota média de aceitação mais baixa $(6,03)$; entretanto, este valor ainda se encontra dentro da faixa de aceitação.

Tabela 6 - Notas de aceitação* para os atributos aparência, sabor, textura, impressão global (IG) e intenção de compra (IC) de bolos formulados com diferentes concentrações de farinha desmucilada de taro. Acceptance scores* for attributes appearance, flavor, texture, overall impression and purchase intent of cakes formulated with different demucilated taro flour concentrations.

\begin{tabular}{cccccc}
\hline Amostras & Aparência & Sabor & Textura & IG & IC \\
\hline $0 \%$ & $7,91^{\mathrm{d}}$ & $7,31^{\mathrm{b}}$ & $7,48^{\mathrm{b}}$ & $7,50^{\mathrm{b}}$ & $4,10^{\mathrm{c}}$ \\
$5 \%$ & $7,43^{\mathrm{cd}}$ & $7,25^{\mathrm{ab}}$ & $7,46^{\mathrm{b}}$ & $7,40^{\mathrm{b}}$ & $3,95^{\mathrm{bc}}$ \\
$10 \%$ & $6,98^{\mathrm{bc}}$ & $6,75^{\mathrm{a}}$ & $7,18^{\mathrm{ab}}$ & $6,93^{\mathrm{a}}$ & $3,68^{\mathrm{ab}}$ \\
$15 \%$ & $6,56^{\mathrm{b}}$ & $6,78^{\mathrm{ab}}$ & $6,86^{\mathrm{a}}$ & $6,80^{\mathrm{a}}$ & $3,51^{\mathrm{a}}$ \\
$20 \%$ & $6,03^{\mathrm{a}}$ & $6,78^{\mathrm{ab}}$ & $6,78^{\mathrm{a}}$ & $6,61^{\mathrm{a}}$ & $3,50^{\mathrm{a}}$
\end{tabular}

${ }^{*}$ Média de 60 provadores. Médias seguidas pela mesma letra, na coluna, não diferem entre si, pelo teste de Tukey, a $5 \%$ de probabilidade

No que diz respeito ao sabor, os bolos que continham $0 \%$ de FDT diferiram dos bolos com 10\% de FDT. As médias das notas de aceitação para o atributo sabor variaram de 6,75 a 7,31, estando entre os escores gostei ligeiramente (6) e gostei muito (8); portanto, assim como para o atributo aparência, estão dentro da faixa de aceitação dos consumidores.

A textura foi um importante atributo avaliado, principalmente por se tratar de bolos formulados com farinha alternativa. As amostras com 0\% e 5\% de FDT diferiram das amostras com $15 \%$ e $20 \%$. As maiores notas foram para os bolos que continham $0 \%, 5 \%$ e
$10 \%$ de FDT, estando elas entre os escores gostei moderadamente (7) e gostei muito (8).

Com relação à impressão global, os bolos formulados com $0 \%$ e $5 \%$ de FDT foram os mais aceitos, seguidos dos bolos com 10\%, $15 \%$ e $20 \%$. Ressalta-se que todos os bolos apresentaram notas médias dentro da faixa de aceitação, ou seja, acima do escore gostei ligeiramente (6).

Para a intenção de compra, que varia em uma escala que parte de certamente não compraria (1) a certamente compraria (5), as amostras com $0 \%$, $5 \%$ e $10 \%$ de farinha desmucilada de taro (FDT) 
receberam as maiores notas, estando entre os escores tenho dúvidas se compraria (3) a certamente compraria (5).

Padilha et al. (2010), ao avaliarem sensorialmente bolos formulados com farinha de yacon, verificaram que a formulação com maior teor de farinha de yacon obteve as maiores notas para os atributos: aroma, gosto doce, maciez, sabor de chocolate e qualidade global; além disso, os autores ressaltaram que os bolos formulados com farinha de yacon possuem a vantagem de conter maior teor de fibra alimentar. Com relação ao bolo em estudo, todos os atributos avaliados (aparência, sabor, textura e impressão global) obtiveram notas médias maiores que 6 , o que os coloca dentro da faixa de aceitação pelos consumidores, com as vantagens nutricionais que a FDT possui: mais fibra alimentar e minerais, como ferro, manganês e zinco.

Para melhor entendimento dos dados referentes à impressão global das amostras de bolos, estes também foram avaliados por meio de um mapa de preferência interno (MPI). As duas primeiras dimensões da análise de componentes principais (ACP) explicaram $73,03 \%$ da variância dos dados experimentais, representando $49,26 \%$ e $23,77 \%$ da variância, respectivamente. Na Figura 4, as amostras foram representadas pelos quadrados, e os consumidores, pelos vetores.

Para entender a relação entre os dados dos consumidores e os sensoriais, o mapa de preferência é um método eficaz, sendo um conjunto de técnicas estatísticas amplamente utilizadas para a otimização de produtos, pela compreensão da relação entre a preferência do consumidor e os dados sensoriais, com o objetivo de identificar os direcionamentos da aceitação (Faye et al., 2006; Greenhoff \& Macfie, 1999).

Como se observa no MPI (Figura 4), as amostras de bolos formulados com $0 \%$ e $5 \%$ foram as mais aceitas pelos consumidores no que se refere à impressão global, e esse resultado corrobora o obtido pela estatística univariada.

$\mathrm{Na}$ Tabela 7, estão apresentados os valores do índice de aceitabilidade dos bolos elaborados com diferentes concentrações de FDT.

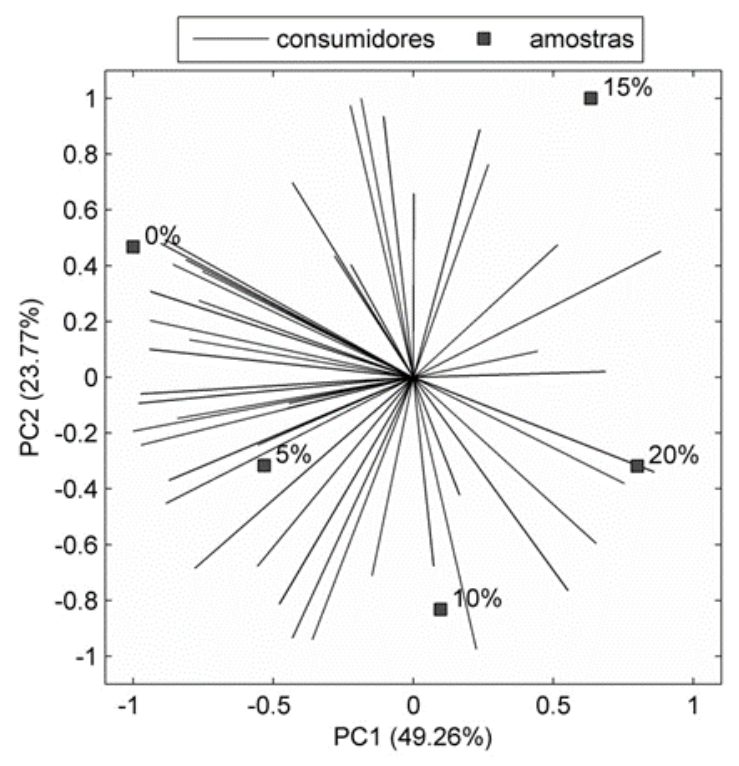

Figura 4 - Mapa de preferência interno (MPI) para as amostras de bolos formulados com diferentes concentrações da farinha desmucilada de taro. Internal Preference Map (IPM) for cake samples formulated with different concentrations of demucilated taro flour.

De acordo com Meilgaard et al. (1991), podem ser considerados favoráveis índices de aceitabilidade $\geq 70 \%$. Todos os bolos em estudo apresentaram boa aceitabilidade com relação aos atributos sabor, textura e impressão. Com relação à aparência, apenas a amostra que continha $20 \%$ de FDT obteve índice menor que $70 \%$.

Moraes et al. (2010), ao avaliarem a aceitação de bolos fabricados com diferentes concentrações de farinha de linhaça, verificaram que a substituição de farinha de trigo por farinha de linhaça em até $30 \%$, no preparo de bolos, constitui uma estratégia útil para viabilizar o consumo de preparações ricas em ingredientes funcionais. A substituição parcial da farinha de trigo pela farinha desmucilada de taro, nos bolos em estudo, também viabiliza o consumo de preparações com maiores teores de fibra, uma vez que a FDT pode ser considerada fonte desse composto.

Tabela 7 - Índice de aceitabilidade das cinco formulações de bolo para os atributos aparência, sabor, textura e impressão global. Acceptability index of the five cake formulations for the attributes appearance, flavor, texture and overall impression.

\begin{tabular}{lccccc}
\hline \multirow{2}{*}{ Atributos } & \multicolumn{5}{c}{ Índice de aceitabilidade ${ }^{1}$ dos bolos $(\%)$} \\
\cline { 2 - 6 } & $0 \%$ & $5 \%$ & $10 \%$ & $15 \%$ & $20 \%$ \\
\hline Aparência & 88 & 83 & 78 & 73 & 67 \\
Sabor & 81 & 81 & 75 & 75 & 75 \\
Textura & 83 & 83 & 80 & 76 & 75 \\
Impressão Global & 83 & 82 & 77 & 76 & 73 \\
\hline
\end{tabular}

${ }^{1}$ Índice de boa aceitação $\geq 70 \%$ (Meilgaard et al., 1991). 


\section{Conclusões}

Nas condições experimentais em que foi realizado o trabalho, conclui-se que, devido à composição química da FDT, com elevados teores de fibra alimentar, amido e microminerais, justifica-se sua utilização na elaboração de bolos.

Bolos podem ser elaborados com a utilização da FDT em até $20 \%$ de concentração, sem alterações expressivas que desfavoreçam o produto final do ponto de vista tecnológico e sensorial. Outros estudos podem ser realizados utilizando maiores concentrações de FDT, sendo também interessante estudo nutricional detalhado dos bolos.

\section{Agradecimentos}

Os autores agradecem à Coordenação de Aperfeiçoamento de Pessoal de Nível Superior (CAPES), ao Conselho Nacional de Desenvolvimento Científico e Tecnológico (CNPq) e à Fundação de Amparo à Pesquisa do Estado de Minas Gerais (Fapemig) pelo apoio financeiro.

\section{Referências}

AACC - American Association of Cereal Chemists Approved Methods of the American Association of Cereal Chemists (method 55-50). 10 $0^{\text {th }}$ ed. St. Paul Minnesota: American Association of Cereal Chemists. 2000.

Aboubakar, Njintang YN, Scher J, Mbofung CMF (2008) Physicochemical, thermal properties and microstructure of six varieties of taro (Colocasia esculenta L. Schott) flours and starches, Journal of Food Engineering 86(2): 294-305.

Andrade LA (2013) Caracterização da mucilagem do taro (Colocasia esculenta) quanto ao poder emulsificante. UFLA (Dissertação em Ciência dos alimentos).

Asm DA, Colmey JC (1973) The role of $\mathrm{pH}$ in cake baking. Baker's Digest 47(1): 36-42.

AOAC - ASSOCIATION OF OFFICIAL ANALYTICAL CHEMISTS (1990) Official methods of analysis. 15th ed. Washington, 1298p.

AOAC - ASSOCIATION OF OFFICIAL ANALYTICAL CHEMISTS (1995) Official methods of analysis. 16. ed. Washington: AOAC.

AOAC - ASSOCIATION OF OFFICIAL ANALYTICAL CHEMISTS INTERNACIONAL (2000) Official methods of analysis of the Association of the Official Analytical Chemists. 17 ed. Gaithersburg: AOAC. v.1. $1170 p$.

Borges JTS, Pirozi MR, Lucia SMD, Pereira PC, Moraes ARF, Castro VC (2006) Utilização de farinha mista de aveia e trigo na elaboração de bolos. Boletim CEPPA 24(1):145-162.
BRASIL (2012) RDC n 54, de 12 de novembro de 2012. Dispõe Sobre o Regulamento Técnico Sobre Informação Nutricional Complementar. Brasília.

Bushuk W (1985) Flour protein:structure and functionality in dough and bread. Cereal Foods Word 30(7): 447-451.

Caruso VR (2012) Mistura para o preparo de bolo sem glúten. Centro Universitário do Instituto Mauá de Tecnologia (Dissertação de Mestrado em Engenharia de Processos Químicos e Bioquímicos).

Cecchi HM (2003) Fundamentos teóricos e práticos em análise de alimentos.UNICAMP. 207 p.

Chavan JK, Kadam SS (1993) Nutritional enrichment of bakery products by supplementation with nonwheat flours. Critical Reviews in Food Science and Nutrition 33(3):189-226.

Duangmal K, Saicheua B, Sueeprasan S (2008) Colour evaluation of freezefried roselle extract as a natural food colorant in a model system of a drink. LWT- Food Science and Technology 41(8):1437-1445.

Dutra OJE, Marchini JS (1998) Ciências Nutricionais. São Paulo: Sarvier, 403p.

Esteller MS, Amaral RL, Lannes SCS (2004) Effect of sugar and fat replacers on the texture of baked goods. Journal of Texture Studies 35(4):383-93.

Esteller, MS, Lannes SCS (2005) Parâmetros complementares para fixação de identidade e qualidade de produtos panificados. Ciência e Tecnologia de Alimentos .25(4): 802-806.

Esteller MS, Zancanaro Júnior O, Lannes SC.S (2006) The effect of kefir addition on microstructure parameters and physical properties of porous white bread. European Food Reserch Technolology 222(12): 26-31.

Fabri ACP (2012) Produção de bolos com baixo teor de sal. Centro Universitário do Instituto Mauá de Tecnologia (Monografia - Pós-graduação em Engenharia de Alimentos).

Faye $P$, Brémaud D, Teillet E, Courcoux P, Giboreau A, Nicod H (2006) An alternative to external preference mapping based on consumer perceptive mapping. Food Quality and Preference 17(7-8): 604-614.

Franco G (1998) Tabela de composição química dos alimentos. São Paulo: Atheneu. 273 p.

Greenhoff K, Macfie HJH (1999) Preference mapping in practice. In: Macfie, HJH, Thomson DM. (Ed.). Measurement of food preferences. New York: Aspen. p137-166. 
Guimarães RR, Freitas MCJ de, Silva VLM da (2010) Bolos simples elaborados com farinha da entrecasca de melancia (Citrullus vulgaris, sobral): avaliação química, física e sensorial. Ciência e Tecnologia de Alimentos 30(2):354-363.

Hecktheuer LHR (1996) Envejecimiento em botella de vinos tintos varietales de tempranillo, bobal $y$ monastrell. Universidad Politecnica de Valencia (Tese de Doutorado em Ciência e Tecnologia de Alimentos).

Hopkins RM, Gracey MS, Hobbs RP, Spargo RM, Yates M, Thompson RC (1997) The prevalence of hookworm infection, iron deficiency and anemia in an aboriginal community in north-west Australia. Medical Journal of Australia 166(5): 241-244.

Huang AS, Titchenal CA, Meilleur BA (2000) Nutrient composition of Hawaiian taro corms and breadfruit. Journal of Food Composition. \& Anal 13(5):859-864.

Huang C, Chen W, Wang C (2007) Comparison of Taiwan paddy- and upland-cultivated taro (Colocasia esculenta L.) cultivars for nutritive values. Food Chemistry 102(1):250-256.

Kaushal P, Kumar V, Sharma HK (2012) Comparative study of physicochemical, functional, antinutritional and pasting properties of taro (Colocasia esculenta), rice (Oryza sativa) flour, pigeonpea (Cajanus cajan) flour and their blends. LWT - Food Science and Technology 48(1):59-68. .

Lawless HT, Heymann H (1998) Sensory evaluation of food. New York: Chapman \& Hall. 819p.

Leonel M, Oliveira MA, Duarte Filho J (2005) Espécies tuberosas tropicais como matérias-primas amiláceas. Revista Raízes e Amidos Tropicais, 1(1):49-68.

Maia SMPC (2007) Aplicação da farinha de maracujá no processamento do bolo de milho e aveia para fins especiais. Universidade Federal do Ceará (Dissertação de Mestrado em Tecnologia de Alimentos).

Malavolta,E, Vitti,GC, Oliveira SA (1997) Avaliação do estado nutricional das plantas: princípios e aplicações. Piracicaba: POTAFÓS. 319p.

Marangoni AL (2007) Potencialidade de aplicação de farinha de Yacon (Polymnia sonchifolia) em produtos à base de cereais. Universidade Estadual de Campinas (Dissertação de Mestrado em Tecnologia de Alimentos).

Meilgaard M, Civille G V, Carr BT (1991) Sensory evaluation techniques. London: CRC Press. 354p.
Miamoto, JBM (2008) Obtenção e caracterização de biscoito tipo cookie elaborado com farinha de inhame (Colocasia esculenta L.). Universidade Federal de Lavras (Dissertação de Mestrado em Ciência dos Alimentos).

Minolta (1994) Precise Color Communication: Color Control from Feeling to Instrumentation. Minolta Co., Ltd., Ramsey, NJ.

Moraes EA, Dantas MIS, Morais DC, Silva CO Da, Castro FAF De, Martino HSD, Ribeiro SMR (2010) Sensory evaluation and nutritional value of cakes prepared with whole flaxseed flour. Ciência e Tecnologia de Alimentos 30(4):974-979.

Nelson NA (1944) A photometric adaptation of Somogy method for the determination of glucose. Journal Biological Chemistry 153(2):135-375.

Nip WK (1997) Taro. In: Smith DS, Cash JN, Nip WK, Hui YH (Eds). Processing vegetable and technology. Pensylvania, USA.Technomic Publishing, pp. 355-387.

Nunes RSC, Pinhati FR, Golinelli, LP, Rebouças TNH, Paschoalin VMF, Silva JT (2012) Polymorphic microsatellites of analysis in cultivars of taro. Horticultura Brasileira 30(1): 106-111.

Osborne DR, Voogt P (1978) The analysis of nutrientes in foods. London: Academic. 47p.

Padilha VM, Rolim PM, Salgado SM, Livera, AS, Andrade SAC, Guerra NB (2010) Perfil sensorial de bolos de chocolate formulados com farinha de yacon (Smallanthus sonchifolius). Ciência e Tecnologia de Alimentos 30(3):735-740.

Santos ES dos, Puiatti M (2002) Cultura do taro (Colocasia esculenta). João Pessoa: EMEPA-PB. 9p.

Schmiele M, Silva LH da, Costa PFP da, Rodrigues RS, Chang YK (2011) Influência da adição de farinha integral de aveia, flocos de aveia e isolado proteico de soja na qualidade tecnológica de bolo inglês. Boletim do Centro de Pesquisa de Processamento de Alimentos 29(1):71-82.

Silva RF, Ascheri J LR, Pereira RGFA, Modesta RDC (2009) Aceitabilidade de biscoitos e bolos à base de arroz com café extrusados. Ciência e Tecnologia de Alimentos 29(4):815-819.

Tavares AS, Pereira J, Guerreiro M.C, Pimenta CJ, Pereira L, Missagia SV (2011) Caracterização físicoquímica da mucilagem de inhame liofilizada. Ciência e Agrotecnologia, 35(5):973-979.

Walkeling I.N, Macfie JH (1995) Designing consumer trials balanced for first and higher orders of carry-over effect when only a subset of k samples from t may be tested. Food Quality and Preference 6(4):299-308. 
Wang J, Rosell CM, Barber CB de (2002) Effect of the addition of different fibres on wheat dough performance and bread quality. Food Chemistry 79(2):221-226.

Ybarra L.M, Costa NMB, Ferreira CLLF (2001) Interação cálcio e ferro: uma revisão. Nutrire: rev. Soc. Bras. Alim. = Nutr. J. Brazilian Soc. Food Nutr 22(1):85-107.
Zárate NAH, Vieira, MC, Minuzzi A (2002) Produtividade de cinco clones de inhame, custos e uso na panificação caseira. Ciência e Agrotecnologia 26(6):1236-12 